\title{
Neonatal cholestasis and focal medullary dysplasia of the kidneys in a case of microcephalic osteodysplastic primordial dwarfism
}

\author{
A Berger, N Haschke, C Kohlhauser, G Amman, U Unterberger, $M$ Weninger
}

\begin{abstract}
We report on a male infant who presented with intrauterine growth retardation, severe postnatal failure to thrive, microcephaly, facial dysmorphism, and skeletal dysplasia. The clinical and radiological findings are consistent with former descriptions of microcephalic osteodysplastic primordial dwarfism (MOPD) type I/III. In addition to previously published features, multiple fractures of the long bones, severe neonatal cholestasis, and histological dysplasia of the kidneys were found. The boy died at the age of 8 months. The new finding of focal renal medullary dysplasia further supports the hypothesis of a basic defect in tissue differentiation in the pathogenesis of this rare condition.

$(\Im$ Med Genet 1998;35:61-64)
\end{abstract}

Keywords: microcephalic osteodysplastic dwarfism; bone fracture; neonatal cholestasis; focal medullary dysplasia of kidney

Based on the thorough review of "bird headed" dwarfism by Majewski and Goecke, ${ }^{1}$ a classification into four different types was established as follows: classical Seckel syndrome and MOPD types I, II, and III, the latter being distinguished from Seckel syndrome by distinct radiological features. According to recent clinical observations, ${ }^{2-5}$ there is now general agreement to consider types I and III as a single entity. ${ }^{67}$

In this report, detailed clinical, radiological, and histological data of an affected infant with MOPD type I/III are presented. Some of our findings have not been described previously.

University Hospital

Vienna, Department of

Pathology,

Waehringerguertel

18-20, 1090 Vienna,

Austria

G Amman

University Hospital

Vienna, Department of

Neuropathology,

Waehringerguertel

18-20, 1090 Vienna,

Austria

U Unterberger

Correspondence to:

Dr Berger.

Received 17 April 1997 Revised version accepted for publication 2 July 1997

\section{Case report}

We report on a male infant, delivered at 31 weeks' gestation by caesarean section because of preterm labour associated with abnormal CTG tracing. The mother of our patient was a 26 year old Turkish woman, married to her 23 year old first cousin. An older sister developed normally. Repeated sonographic examinations from 21 weeks of gestation onwards showed severe intrauterine growth retardation. Amniocentesis performed at 23 weeks showed a normal karyotype and normal amniotic alphafetoprotein.

Birth weight was $786 \mathrm{~g}$ ( $-3 \mathrm{SD})$, length 32 $\mathrm{cm}(-3.4 \mathrm{SD})$, and head circumference 21.7 $\mathrm{cm}$ (-6 SD). APGAR scores were 5 and 7 at one and five minutes, respectively. The child had mild respiratory distress syndrome requiring ventilatory support for only one hour.

The following abnormalities were noted (fig 1): microcephalic dwarfism with a sloping forehead, small anterior fontanelle, sparse eyebrows, large eyes, beaked nose, thick lips, moderate micrognathia, short neck, broad hands with marked tapering of fingers, clinodactyly of the 5th fingers, short and broad feet with prominent heels, bilateral cryptorchidism, and dry, slightly hyperkeratotic skin.

On cranial ultrasound, partial agenesis of the corpus callosum and minimal cortical gyral differentiation were noted. The renal tract and heart were normal on ultrasound. A normal male karyotype was confirmed.

Thorough investigations for intrauterine infections and metabolic and endocrine disorders were negative.

The clinical course was complicated by recurrent infections from the first week of life onwards. The differential count was extremely shifted to the left (immature to total neutrophil ratio up to 0.9 ), even in intervals without clinical signs of infection. No specific immunological disorder was found. Bone marrow aspiration of the tibia was unsuccessful. The procedure was not repeated because of generalised osteoporosis and subsequent multiple fractures.

Besides recurrent infections, the most striking clinical findings were hepatosplenomegaly and cholestasis. Repeated serological and metabolic investigations were negative and a biopsy of the liver showed non-specific pathology (giant cell hepatitis, fibrosis, cholestasis) without evidence of storage disease.

Growth and weight gain were poor and remained far below the 3rd centile; psychomotor development was severely retarded.

At the age of 7 months the boy developed persistent hypokalaemia, hypophosphataemia, and hypocalcaemia with urinary loss of protein, glucose, potassium, calcium, and phosphate. Despite repeated courses of antibiotic treatment the child died of pneumonia and sepsis at the age of 8 months.

\section{RADIOLOGICAL FINDINGS}

There was microcephaly and generalised osteoporosis. The spine showed mild platyspondyly. The pelvis was dysplastic with rounded, hypoplastic iliac wings and nearly horizontal, irregular acetabular roofs (fig 2A). Both femora showed enlarged proximal metaphyses (fig 2B). There was a total lack of epiphyseal ossification. 

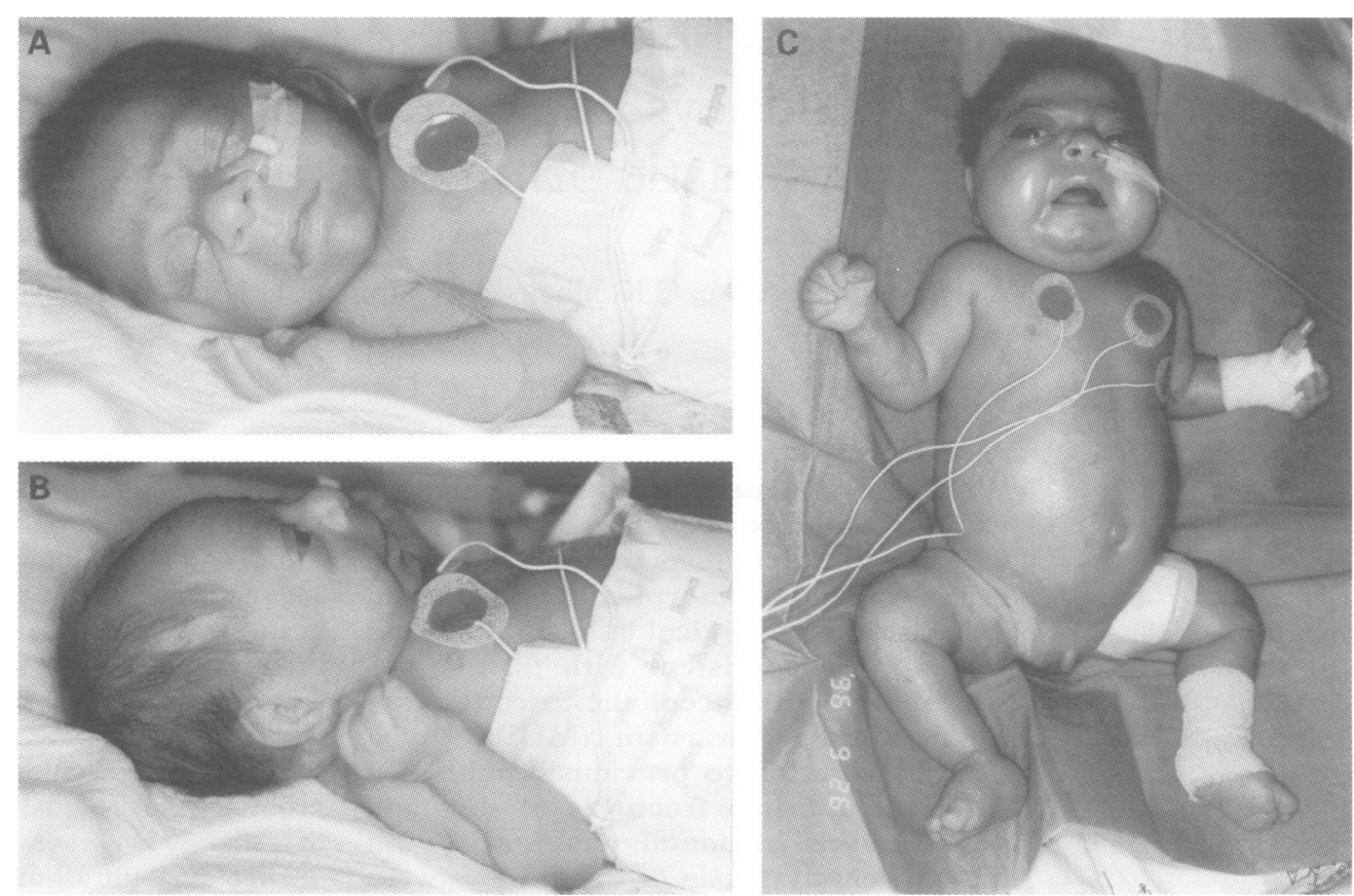

Figure 1 The proband aged 2 months $(A, B)$ and 8 months $(C)$. Note microcephaly, beaked nose, tapering of fingers, dorsal lymphoedema of the feet, and hypoplastic genitalia.

At the age of 7 months the first bone fracture was noted on routine $x$ ray. Following the initial proximal femoral location, numerous additional lesions involving the distal femoral, proximal tibial, and proximal humeral bones and the ribs were observed (fig 2C). Postmortem studies confirmed these findings.

NECROPSY MACROSCOPIC FINDINGS

The spongiosa of the long bones was hypoplastic and porotic. Massive hepatosplenomegaly was noted. The liver showed fibrosis and cholestasis. There was cardiac dilatation and marked gas distension of the gut. The kidneys were small with icteric nephropathy. The thymus appeared involuted.

The brain was small (118 g) and abnormally shaped with hypoplastic frontal lobes. Gyration was plump but symmetrical. Partial agenesis of the corpus callosum (fig 3A) and a synthalamus were found. The cerebellar vermis was hypoplastic, with distorted configuration.
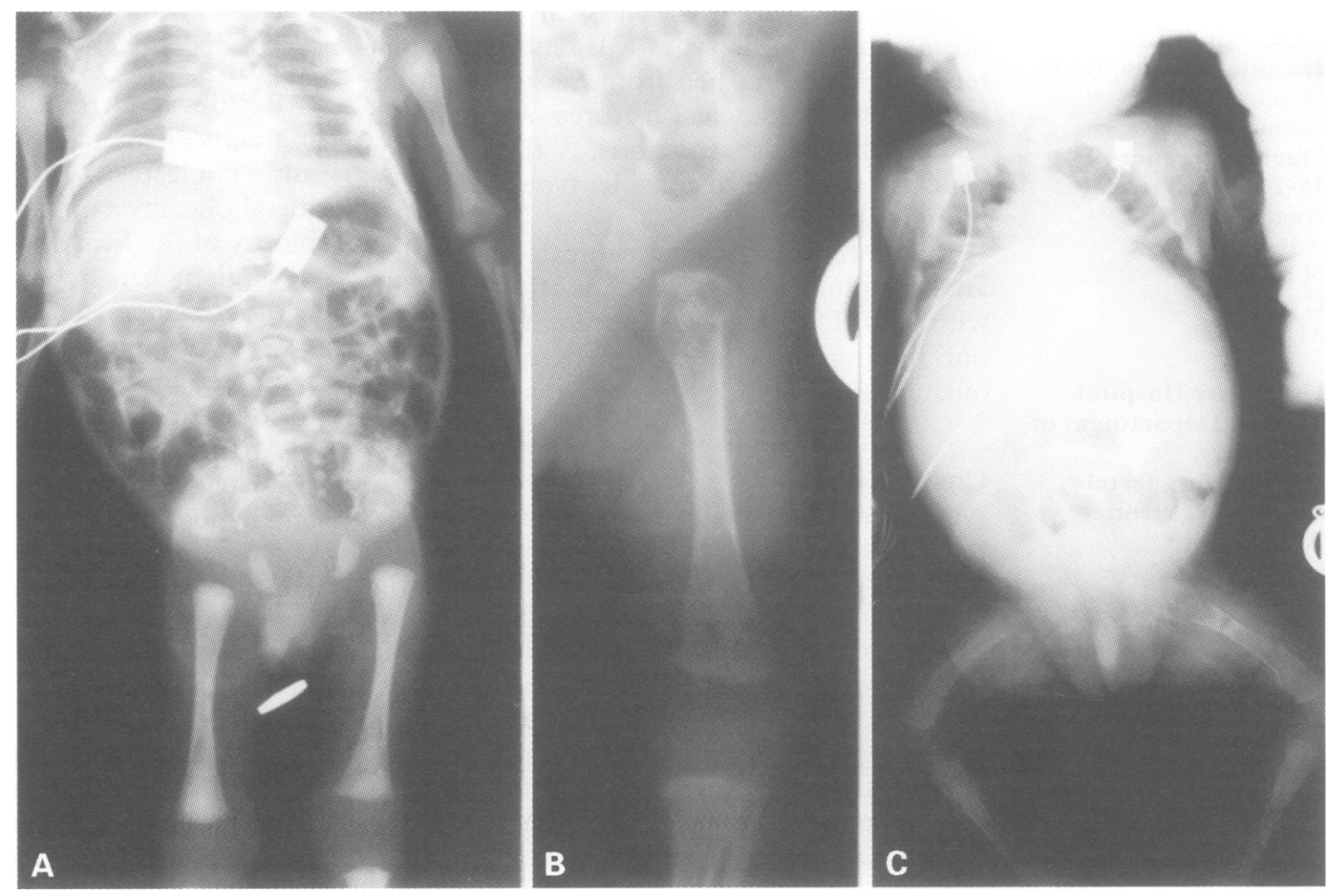

Figure 2 Radiographs of patient at birth (A), 4 months $(B)$, and 7 months $(C)$. (A) Note dysplastic pelvis with nearly horizontal acetabular roofs and general lack of epiphyseal ossification. (B) Note enlarged proximal femoral metaphysis and proximally long fibula. (C) Note severe generalised osteoporosis and fractures of the lefi proximal humerus, both proximal and distal femoral bones, and proximal tibial bones. 

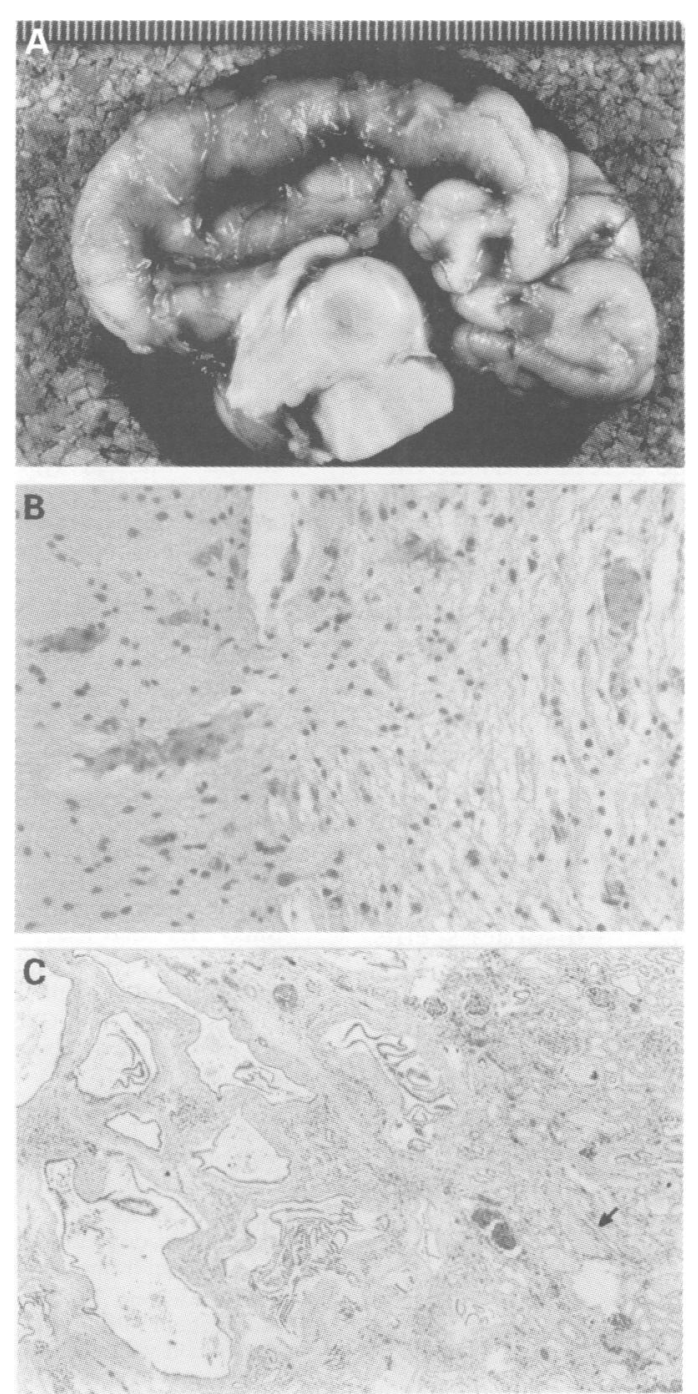

Figure 3 Brain of patient $(A)$ and photomicrographs of brain $(B)$ and kidney $(C)$. (A) Median sagittal section through the brain. Note partial agenesis of the corpus callosum and hypoplastic frontal lobe. (B) High power view of the cerebral cortical surface. The cortical neuronal architecture is disturbed; note the heterotopic glioneuronal tissue overlying the cortex (right side) (HEE). (C) Kidney showing dilated and cystic medullary nephron segments (left side) and some mildly ectatic tubules (arrow) in the adjacent cortex $(H \mathcal{E} E)$.

\section{MICROSCOPIC FINDINGS}

Liver

Severe parenchymal alteration was found which was essentially non-specific and in keeping with remnants of a so-called giant cell hepatitis progressing to cirrhosis. There was centrilobular accentuation of parenchymal loss, accompanied by extensive cellular, canalicular, and ductular cholestasis with marked proliferation of portal bile ducts.

\section{Kidneys}

The most striking finding was the presence of focal medullary dysplasia with secondary changes in the corresponding cortex, the latter presenting with ectatic tubules and tubular as well as glomerular cysts (fig $3 \mathrm{C}$ ). In addition, rather large foci of interstitial extramedullary haematopoiesis were found.

Bones (ribs, tibia, and femur)

There was a striking reduction in cancellous bone mass with significantly decreased osteo- blastic activity. The marrow cavity was enlarged and filled with haematopoetic cells. Irregular intracartilaginous ossification was seen with diminished height of cartilage cell columns and disturbed ossification.

\section{Brain}

The cerebral cortex was abnormally wide with a slightly disturbed lamination pattern. There were heterotopic neurones in the molecular layer of the cortex as well as irregular clusters of neurones in the white matter. Overlying the cortex was a layer of heterotopic glioneuronal tissue (fig 3B), predominantly on the medial surface and dorsal convexity of the hemispheres. The hippocampus was malrotated. Cerebellar folia were dysplastic in some areas with multiple micropolygyric convolutions.

\section{Discussion}

Majewski et $a l^{189}$ delineated three types of microcephalic osteodysplastic primordial dwarfism differing from Seckel syndrome on the basis of radiological findings. Types I and III are now considered to be a single entity with a possible age dependency of the $x$ ray findings. ${ }^{2-7}$ It was suggested that the term "type III" should be abandoned altogether, ${ }^{5710}$ leaving only two types, type I, including all cases formerly reported as type I or type III plus the cases published as "cephaloskeletal dysplasia", $11-13$ and type II. ${ }^{8}$ Consequently, the term "type III" became free. Confusingly, in 1992, Majewski ${ }^{14}$ again used this term to describe the syndrome of Caroline Crachami, a "new" entity of osteodysplastic primordial dwarfism.

Like previous cases, our patient died during an episode of respiratory infection. We observed extremely immature cells in the peripheral blood, even in intervals without clinical infection. Taybi and Linder ${ }^{11}$ reported a similar shift to the left in the differential of their second case. However, the reported blood count was taken shortly before death from pneumonia. Unfortunately, an attempt at bone marrow aspiration showed only peripheral blood cells. Based on reports of infections as the primary cause of death in most cases that survived for a longer time $2459-11$ and supported by excessive extramedullary haematopoesis involving the liver, spleen, kidneys, and testes (postmortem findings) in our case, we suggest that abnormalities in haematology and perhaps immune response, although not confirmed in our case, are part of this syndrome. In future, bone marrow examination at an earlier stage of disease could provide further important information.

A feature not formerly described was the marked cholestasis and hepatosplenomegaly of our patient. No other reported case of MOPD has shown this pathology. Taybi and Linder ${ }^{11}$ reported jaundice without hepatosplenomegaly. In our case, histological examination showed no specific alterations. In addition, repeated serological studies were not conclusive, leaving only parenteral nutrition and recurrent infections as possible explanations for this clinical finding. Only recently, Eason et $a l^{10}$ reported the previously unrecognised feature of renal tubular leakage in a case of 
MOPD I/III. Our patient developed analogous symptoms in his seventh month of life including hypokalaemia, hypophosphataemia, and hypocalcaemia with marked urinary loss. While necropsy was not performed in the case of Eason et al, ${ }^{10}$ Winter et al described focal dilatation of the proximal renal tubules and fibrin deposits occluding the capillaries of a few glomeruli in their patient. With respect to these rather non-specific findings, the presence of focal medullary dysplasia in our case appears to be of particular interest, as it provides additional evidence that a basic defect of tissue differentiation might be primarily involved in the pathogenesis of this condition.

Multiple fractures of the long bones and ribs as observed in our case have not been described previously. We assume that they result from the renal problems and the primary disease. Eason et $a l^{10}$ also described marked osteoporosis in their case. Histology of the bone was similar to previous descriptions. ${ }^{311}$ Our neuropathological findings showing a profound disturbance of cerebral development are also similar to former reports. ${ }^{31116}$

The clinical variability of this disorder seems to be broad; additional findings in our patient were neonatal cholestasis and multiple fractures of the long bones. The pathogenesis is unknown, but we propose a basic defect in cell proliferation and tissue differentiation. This hypothesis is further underlined by our finding of focal medullary dysplasia of the kidneys. Autosomal recessive inheritance is likely, based on reports of parental consanguinity ${ }^{11}{ }^{15}$ (our case) and incidence among sibs of different sex. The gene responsible remains to be defined.

The authors are grateful to Dr S Fang Kircher for fruitful discussion and to Professor W Ponhold for diagnostic evaluation of the radiographs. We especially thank Professor Dr A Pollak for thorough revision of the manuscript.
1 Majewski F, Goecke T. Studies of microcephalic primordial dwarfism. I. Approach to a delineation of the Seckel syndrome. Am $\mathcal{F}$ Med Genet 1982;12:7-21.

2 Winter RM, Wigglesworth J, Harding BN. Osteodysplastic primordial dwarfism: report of a further patient with manifestations similar to those seen in patients with types I and III. Am $\mathcal{Y}$ Med Genet 1985;21:569-74.

3 Haan EA, Furness ME, Knowles S, et al Osteodysplastic primordial dwarfism: report of a further case with manifestations similar to those of types I and III. Am $\mathcal{F}$ Med Genet 1989;33:224-7.

4 Meinecke P, Schaefer E, Wiedemann HR. Microcephalic osteodysplastic primordial dwarfism: further evidence for identity of the so-called types I and III. Am 7 Med Genet identity of the so-c
$1991 ; 39: 232-6$.

5 Meinecke P, Passarge E. Microcephalic osteodysplastic primordial dwarfism type I/III in sibs. $f$ Med Genet 1991;28:795-800.

6 Taybi H. Cephalo-skeletal dysplasia and microcephalic osteodysplastic primordial dwarfism. Pediatr Radiol 1992; 22:476.

7 Meinecke P, Passarge E. Reply to Dr Taybi. Am $\mathcal{F}$ Med Genet 1992;43:629.

8 Majewski F, Ranke M, Schinzel A. Studies of microcephalic primordial dwarfism. II. The osteodysplastic type II of primordial dwarfism. Am $\mathcal{F}$ Med Genet 1982;12:23-35.

9 Majewski F, Stoeckenius M, Kemperdick H. Studies of microcephalic primordial dwarfism. III. An intrauterine dwarf with platyspondyly and anomalies of pelvis and dwarf with platyspondyly and anomalies of pelvis and
clavicles - osteodysplastic primordial dwarfism type III. $\mathrm{Am}$ clavicles - osteodysplastic primc

10 Eason J, Hall CM, Trounce JQ. Renal tubular leakage complicating microcephalic osteodysplastic primordial dwarfism. 7 Med Genet 1995;32:234-5.

11 Taybi H, Linder D. Congenital familial dwarfism with cephalo-skeletal dysplasia. Radiology 1967;89:2754-81.

12 Thomas PS, Nevin NC. Congenital familial dwarfism with cephaloskeletal dysplasia (Taybi-Linder syndrome). Ann Radiol 1976;19:187-92.

13 Lavollay B, Faure C, Filipe G, Branca G, Huet de Barochez Y. Nanisme familial congénital avec dysplasia céphalosquelettique (syndrome de Taybi-Linder). Arch Fr Pediatr 1984;41:57-60.

14 Majewski F. Caroline Crachami and the delineation of osteodysplastic primordial dwarfism type III, an autosomal recessive syndrome. Am $\mathcal{F}$ Med Genet 1992;44:203-9.

15 Van Maldergem L, Gillerot Y, Godhaird M, Nemec E, Koulischer L. Primordial osteodysplastic dwarfism type I in association with corneal clouding: evidence for autosomal recessive inheritance. Clin Genet 1990;38:359-61.

16 Kozlowski K, Donovan T, Masel J, Wright RG. Microcephalic, osteodysplastic, primordial dwarfism. Australa Radiol 1993;37:111-14. 